\title{
BMJ Open High HIV seroprevalence, rectal STIs and risky sexual behaviour in men who have sex with men in Dar es Salaam and Tanga, Tanzania
}

\author{
Michael W Ross, ${ }^{1}$ Joyce Nyoni, ${ }^{2}$ Hycienth O Ahaneku, ${ }^{1}$ Jessie Mbwambo, ${ }^{3}$ \\ R Scott McClelland, ${ }^{4}$ Sheryl A McCurdy ${ }^{1}$
}

To cite: Ross MW, Nyoni J, Ahaneku HO, et al. High HIV seroprevalence, rectal STIs and risky sexual behaviour in men who have sex with men in Dar es Salaam and Tanga Tanzania. BMJ Open 2014;4 e006175. doi:10.1136/ bmjopen-2014-006175

- Prepublication history for this paper is available online. To view these files please visit the journal online (http://dx.doi.org/10.1136/ bmjopen-2014-006175).

Received 23 July 2014 Accepted 6 August 2014

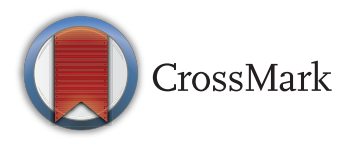

${ }^{1}$ Center for Health Promotion and Prevention Research, School of Public Health, University of Texas, Houston, Texas, USA

${ }^{2}$ Department of Sociology and Anthropology, University of Dar es Salaam, Dar es Salaam, Tanzania

${ }^{3}$ Department of Psychiatry and Mental Health, Muhimbili University of Health and Allied Sciences, Dar es Salaam, Tanzania

${ }^{4}$ University of Washington/ University of Nairobi Mombasa STI Laboratory, Seattle, Washington, USA

Correspondence to Dr Michael Ross;

Michael.W.Ross@uth.tmc.edu

\section{ABSTRACT}

Objectives: To assess HIV and sexually transmitted infection (STI) prevalence and associated risk factors in men who have sex with men (MSM) in two cities in mainland Tanzania.

Methods: We conducted respondent-driven sampling of 300 MSM in Dar es Salaam and Tanga.

Results: In Dar es Salaam, 172 (86\%) men (median age 23, IQR 21-28) consented to HIV/STI testing, and $30.2 \%$ were HIV seropositive. Only five reported a previous positive HIV test: $>90 \%$ were new HIV detections. $2.5 \%$ were syphilis-exposed and none hepatitis B positive, but $21.4 \%$ had a curable STI. Over $90 \%$ of the gonorrhoea and chlamydia was rectal. In Tanga, $11.1 \%$ of MSM were HIV seropositive, $8 \%$ hepatitis B positive and $0 \%$ were syphilis-exposed, with $4.4 \%$ having a curable STI. Predictors of HIV infection were number of MSM known, city, identifying as gay and having first sex with a man. Predictors for STIs were recent unprotected receptive anal intercourse, and number of MSM seen in the last month. $30 \%$ of the sample reported that they sold sex. There was no significant association between HIV and STI infection.

Conclusions: HIV and STI rates were substantially lower in MSM in a provincial city than in a large metropolis and rates appear to depend on larger numbers of MSM known. Most HIV detected were new cases, and there was a high burden of asymptomatic curable rectal STIs (>1 in 5 MSM). Owing to stigma, MSM may not report homosexuality and thus not have rectal STIs treated. High need for tailored HIV testing and STI screening and treatment of MSM in Tanzania is apparent.

\section{INTRODUCTION}

There are significant populations of men who have sex with men (MSM) in East Africa. In Mombasa, Kenya, a port city about 200 miles north of Dar es Salaam, Geibel et $a l^{1}$ identified 77 potential MSM contact locations and calculated that 739 men sell

\section{Strengths and limitations of this study}

It is the first study of mainland Tanzanian men who have sex with men (MSM) with behavioural and HIV and sexually transmitted infection prevalence data.

- The study samples MSM from a large metropolitan area and a smaller provincial city.

- The study provides prevalence data for gonorrhoea and chlamydia from urethral and rectal samples, HIV, hepatitis B infection and past or present syphilis infection.

- The data are cross-sectional.

- The data may not be generalisable outside East Africa or to non-urban settings.

sex to other men in and around Mombasa (population about 900000 ). Sanders et $a l^{2}$ recruited 285 MSM in Mombasa. There was a high level of HIV-1 infection in the exclusive MSM (43\%) compared with the MSM who also had sex with women $(12 \%)$ (Kenyan general population, $6.1 \%)$. Significant adjusted predictors for HIV infection were (reference group insertive anal intercourse only): receptive anal intercourse (RAI) only $(\mathrm{OR}=3.9)$ and both insertive and RAI $(\mathrm{OR}=8.0)$; being exclusively MSM ( $\mathrm{OR}=6.3)$; having peri-anal condylomata on examination ( $\mathrm{OR}=5.1)$; and, in the 18-24 age group, an OR equivalent to 1.10 /year of age.

A study of MSM in Malawi, Namibia and Botswana $^{3}$ in 2008 indicated that HIV seroprevalence increased with age, with an overall prevalence of $17.4 \%$, with $5.1 \%$ denied healthcare based on their sexuality and $18.5 \%$ afraid to seek health services for the same reason. While HIV seroprevalence was $8.3 \%$ in those aged under 24, it was $35.7 \%$ for those aged over 30. Price et at reviewed MSM studies in Nairobi and Cape Town and found that predictors of HIV acquisition included report of genital ulcer 
$(\mathrm{HR}=4.5)$, not completing secondary education $(\mathrm{HR}=3.4)$, RAI $(\mathrm{HR}=8.2)$ and paying for sex $(\mathrm{HR}=0.2)$.

In a cohort in coastal Kenya, Sanders et a $\bar{l}$ reported that $75 \%$ of symptomatic and $12 \%$ of asymptomatic MSM had rectal chlamydia or gonorrhoea $(18 \%$ of those with any history of receptive anal intercourse). In their sample, HIV incidence was 8.6/100 person-years, with one-third of exclusively MSM acquiring HIV within a year of study enrolment.

HIV studies of MSM in mainland Tanzania have not been published, although Dahoma et al and Johnston et $a l^{7}$ have found that for MSM on Zanzibar island there is high overlap between MSM risk behaviour and injecting drug use (IDU) (14\%), with IDU-MSM more likely to have paying partners and MSM having generally high levels of paying partners and risk behaviour despite high awareness of HIV. Dahoma et at report HIV prevalence of $12.3 \%$ in MSM (compared with about $0.5 \%$ of adult men in the general Zanzibar population) associated with hepatitis $\mathrm{C}$ infection and paying partners. Our earlier studies of MSM in Dar es Salaam indicated nearly 100 MSM-related meeting places widely distributed across the city. ${ }^{8}$ As there are few data on prevalence of HIV in the context of sexually transmitted infection (STI) co-infection in African MSM, we examined HIV risk behaviours, seroprevalence of syphilis and urethral and rectal chlamydia and gonorrhoea prevalence in MSM in a major and a provincial city in north-east Tanzania.

\section{METHODS}

Study design, sampling and recruitment

Data were collected in 2012 (Dar es Salaam) and 2013 (Tanga) from a cross-sectional survey of 200 MSM in Dar es Salaam and 100 in Tanga, Tanzania. Dar es Salaam is the major city in Tanzania, with a metropolitan area population of about 3 million, while Tanga is a provincial city in the northeast, midway between Dar es Salaam and Mombasa, with a metropolitan population of about 280 000. Data were collected from 300 MSM using RDS (Respondent Driven Sampling). RDS is a research method that involves snowball sampling where a researcher can use 'seed' individuals to refer those they know and in turn these individuals refer those they know, using a predetermined number of coupons, throughout a network. ${ }^{9}$ RDS was used during data collection for two main reasons: first, we were dealing with a closed community which cannot be reached easily and this method helped us to reach them since they recruited themselves. Second, homosexual behaviour is illegal and highly stigmatised in Tanzania and it was not easy for us to reach MSM openly so we need a reference from other members from the target population to gather information. We identified five seeds in each city, who were different ages and from different areas of each city for better representativeness, to recruit the first wave of participants. Each seed or referrer was given three coupons to recruit three members who they knew were
MSM. Coupons had unique identification numbers linking coupons to the referring seed. Characteristics of those recruited as part of the waves were continually compared to the characteristics of seeds to determine when the sample reached equilibrium for age and education. As we were about to close any seed we reduced the number of coupons to two and the last respondents in each seed were not given any coupon. Our desired sample size of 200 in Dar es Salaam and 100 in Tanga was reached after an average of seven waves. Equilibrium for education and age was also reached after seven waves.

Based on HIV rates reported in MSM in Mombasa of $40 \%$ in exclusively gay men and $10 \%$ in bisexuals, ${ }^{2}$ with $40 \%$ in one group and $20 \%$ in the other and a power of $80 \%$, we would need a minimum of 80 in each group. We anticipated that the differences between groups may be smaller (in the order of $25 \%$ in one group to $13 \%$ in the other) than in Mombasa and probably vary by city, so in order to obtain $80 \%$ power in each city, we calculated a total sample of 310 .

\section{Procedure}

Eligible participants underwent a self-administered interview using a structured questionnaire on laptop with some open-ended questions, assisted by a research assistant. Prior to interview, participants were fully informed regarding the purpose of the interview, the study's rationale and the benefits/risks of participation. To protect participants' identity and confidentiality, oral informed consents were obtained in Swahili or English (the two official languages of Tanzania), as the participant preferred. The interview took about 30-40 min to administer. If participants had difficulty in understanding item(s), research assistants who were graduates and trained in research interviewing read or explained the item(s) in a manner consistent with the item's meaning. For participants who could not read, a research assistant read all questions to the participants. The interviews took place in private at a house rented for the project or in a prearranged alternative safe location. Participants could choose to take the interview in Swahili or English. For the Swahili version of the questionnaire, the accuracy of the translations and its content validity was assessed by a panel of native Swahili-speaking experts. After translation into Swahili and back-translation into English, the questionnaire was pilot-tested with five MSM for comprehension, clarity and response range and modified as appropriate. Each participant and the referrer received an equivalent of US\$2.75 in compensation for transportation.

The study used an 8-item short version of the Internalised Homonegativity (IH) 'Reactions to Homosexuality Scale'. ${ }^{10}$ This IH scale has been validated among MSM in Sub-Saharan Africa (SSA) and comprises factors including personal comfort with homosexuality, social comfort with gay men and public identification as gay $^{11}{ }^{12}$ with a 6-point Likert-type response from 
$1=$ strongly disagree to $6=$ strongly agree. We used the Patient Health Questionnaire-9 (PHQ-9), a validated instrument measuring the nine diagnostic criteria for DSM-IV depressive disorders. ${ }^{13}$ This 9 -item scale measures depression diagnosis and severity. It has been extensively used in SSA and validated in Swahili. ${ }^{14}$

HIV-1 was tested by two rapid tests, Determine, Abbott Laboratories, Chicago, Illinois, USA; and Unigold (Trinity Biotech, Bray, Ireland) to confirm a positive test. Syphilis antibody was tested using DetermineTP Rapid Syphilis Assay and hepatitis B was tested for hepatitis B surface antigen (HBsAg) using Determine HBsAg assay (both Inverness Medical Innovations, Waltham, Massachusetts, USA). Chlamydia and gonorrhoea were tested at the University of Washington/University of Nairobi Mombasa STI Laboratory with a first-pass urine sample and an anal swab using APTIMA Combo2 (Hologic Gen-Probe, San Diego, California, USA). Pretest and post-test HIV counselling was provided according to Tanzanian national guidelines and all HIV seropositive men were referred on to the Muhimbili Hospital HIV Centre in Dar es Salaam or the Bombo Regional Hospital in Tanga. Syndromic STI examination and treatment was provided at the time of data collection.

\section{ANALYSES}

Data were analysed using SPSS V.21. Categorical data were analysed using $\chi^{2}$ analyses with Yates correction for discontinuity where appropriate and interval or ratio data using t test with pooled variance estimates unless Levene's F was significant, when separate variance estimates were used. Logistic regressions utilised Hosmer and Lemeshow's criterion for inclusion of independent variables. All tests were 2-tailed with significance set at the 5\% level. For logistic regression, continuous variables were split at the median and analysed as binary categories. As Heckathorn ${ }^{15}$ advises, unadjusted data were used for analyses of associations. As RDS obtains a random sample of networks, ${ }^{16}{ }^{18}$ we used a combination of parametric and non-parametric tests as appropriate.

\section{RESULTS}

The sample comprised Tanzanian nationals, disproportionately well educated, median age 23 (IQR 21-28) for Dar es Salaam and Tanga. The majority was never married and nearly a third reported selling sex as their major source of income (table 1). Of the 200 respondents in Dar es Salaam, 172 (86\%) consented to an HIV test. Of those, $52(30.2 \%)$ were HIV seropositive. Only 5 of the total 62 positives in both cities reported a previous positive HIV test: thus, $91.9 \%$ of those who tested positive were new detections. Only $5(1.9 \%)$ of those who tested had evidence of past or present syphilis infection (all in Dar es Salaam) and $8(3.1 \%)$ hepatitis B positive (all in Tanga) (table 2). The burden of HIV/STIs was markedly higher in Dar es Salaam than Tanga. Of those with a rectal STI, only $12.5 \%$ reported ever having an anal discharge (compared with $9 \%$ of those without a rectal STI, Yates $\chi^{2}=0.44, \mathrm{df}=1, \mathrm{p}=0.83$ ). There was no significant relationship between HIV status and STI status $\left(\chi^{2}=0.53, \mathrm{df}=1, \mathrm{p}=0.47\right)$. The great majority of respondents $(87 \%)$ reported they were circumcised and there were no differences in HIV infection by circumcision status. HIV infection differed significantly between the exclusive MSM (sex only with men in the past 5 years, $38.5 \%$ ) and men who had sex with men and women $($ MSMW $)$ in that time $\left(10.7 \%, \chi^{2}=27.9, \mathrm{df}=1, \mathrm{p}=0.001\right)$, but there were no significant differences in urethral and anal STI rates between these 2 groups. RAI without a condom with one of the last three partners was associated with both HIV infection (tables 3 and 4) and a rectal STI $\left(29.2 \%\right.$ vs $\left.11.2 \%, \chi^{2}=10.5, \mathrm{df}=1, \mathrm{p}=0.001\right)$.

Sexual positioning preference taken over the last three partners was no anal sex, 24.5\%; receptive anal only, 33\%; insertive anal only, 32.5\%; and versatile (insertive and receptive), $10 \%$. There was no significant relationship between those who considered themselves exclusively gay $(n=175)$ and bisexual $(n=125)$ on partner numbers in the past 6 months $(8.1 \pm 13.2$ vs $7.6 \pm 6.9$, $\mathrm{t}=0.37, \mathrm{p}=0.71)$. Almost all the respondents knew that receptive $(94.3 \%)$ and insertive $(92.9 \%)$ anal sex without a condom could spread HIV.

Tables 5 and 6 illustrates predictors of HIV and STI infection. Size of city, number of gay men known, selfidentifying as gay (as opposed to bisexual) and having a man as the first sexual partner all significantly predicted HIV infection. For STI infection, receptive anal sex without condoms with 1 of the last 3 sexual partners, size of city, number of gay men known and number of those gay men seen over the last month were all significant predictors.

\section{DISCUSSION}

This sample is the first collected for MSM in mainland Tanzania which includes HIV, hepatitis B, syphilis serostatus, and urethral and rectal chlamydia and gonorrhoea. The HIV rate of over $30 \%$ for those tested in Dar es Salaam is very high, comparable to the $35 \%$ HIV rate of IDUs tested in Dar es Salaam. ${ }^{19}$ Syphilis infection rates, which could include evidence of previous or present infection, were low at $2.5 \%$ (consistent with Zanzibar MSM data, which also utilised an antibody detection test), and no hepatitis $B$ infection was detected. In Tanga, in contrast, HIV and STI rates were lower, a third that of Dar es Salaam, suggesting that a much smaller provincial city may have significantly lower (but still unacceptably high) rates in MSM compared with the major metropolis. This is probably due to smaller networks of MSM, and low levels of travel of MSM between provincial cities and the major metropolis. High HIV rates are likely an indication of the lack until very recently of HIV interventions carried out in 
Table 1 Demographic variables

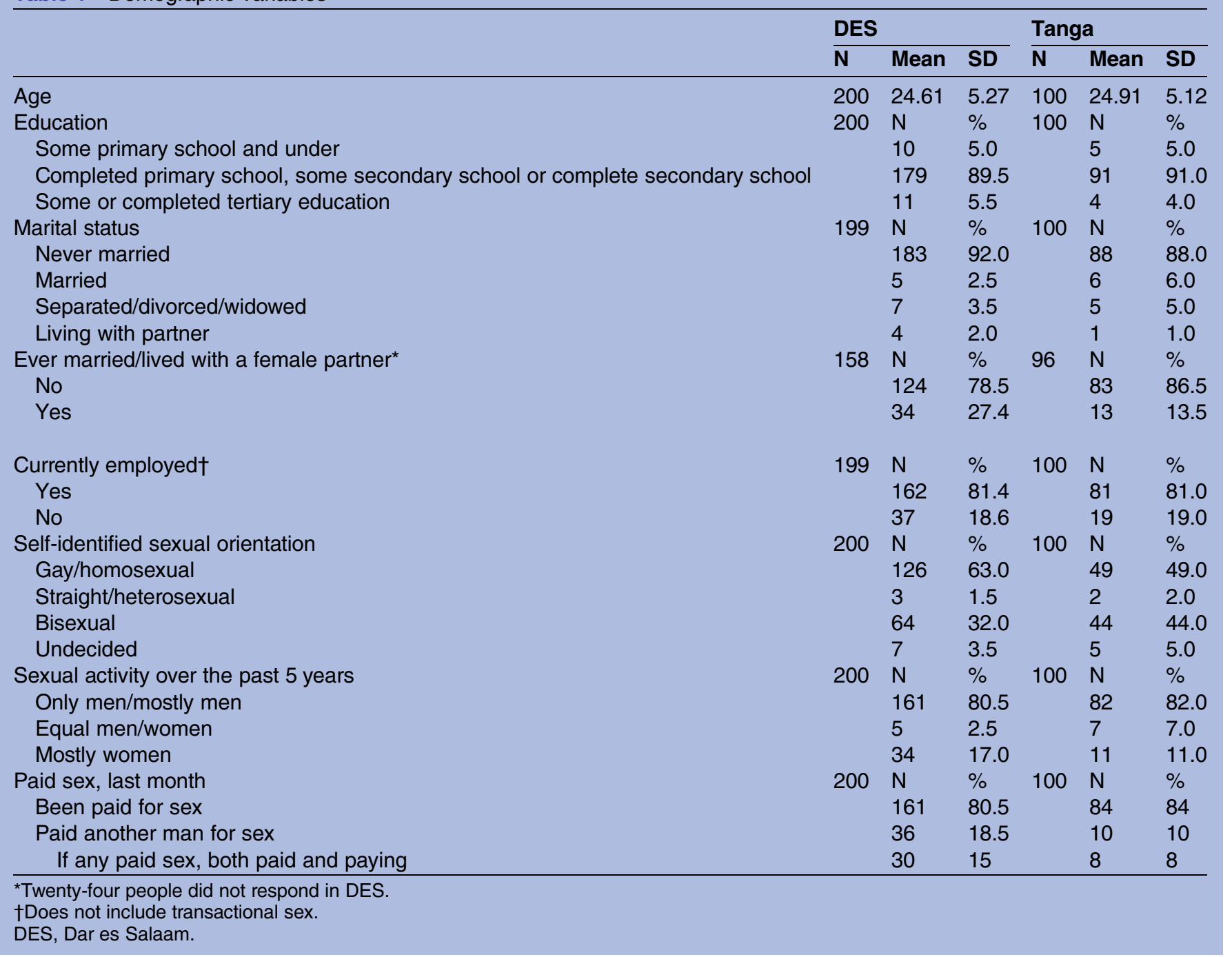

MSM in mainland Tanzania and probably represent the approximate rate in an un-accessed MSM population.

That $>90 \%$ of the HIV seropositives were newly detected cases underscores the fact that this is a largely untested population, or at least not tested for some time. Only $14 \%$ of respondents did not want to wait for results and this suggests that most MSM do want to know their test results and, by implication, prevent HIV spread and access treatment. This is an accessible and undertested population who responded positively to community outreach. However, it is likely that they will require some help to access and trust treatment services for HIV and STIs given the stigma associated with homosexuality.

In contrast with MSM in Zanzibar (45 miles offshore from Dar es Salaam: Zanzibar City population about 220000 ) who reported $12.3 \%$ HIV prevalence and predictors of injecting drugs in the past 3 months, hepatitis $\mathrm{C}$ infection and being paid for sex in the past year, ${ }^{6} 7$ the Dar es Salaam sample had 2.5 times the HIV prevalence in Zanzibar and quite different predictors. These predictors were receptive anal sex and lifetime numbers of sex partners. There are clearly different dynamics in geographically close MSM communities associated with HIV infection in East Africa, and a need to tailor prevention programmes to local contexts. In Dar es Salaam, MSM also had an HIV rate some 2.5 times that of the general population. Nearly a third reported selling sex as their major source of income, and this figure was very similar to our study of MSM 5 years previously in Dar es Salaam ${ }^{20}$ and our later data. ${ }^{21}$ These data are consistent with those of Sanders et $a \bar{p}^{22}$ a few hundred miles north in coastal Kenya, with significant levels of rectal gonorrhoea and chlamydia in MSM and similar rates of selling and buying sex, although we did not replicate an association between STIs and HIV infection. We also found, like Sanders et al, significantly higher differences in HIV infection rates in exclusive MSM compared with MSM who also had sex with women and in those MSM who reported they had unprotected RAI with one of their last 3 sexual partners. Despite lower prevalence in MSMW and in a provincial city, these rates in a smaller city and in MSMW are still substantial: as MSMW are a 
Table 2 STI and HIV prevalence among MSM in DES and Tanga

\begin{tabular}{|c|c|c|c|c|c|c|}
\hline \multirow[b]{2}{*}{ Variable } & \multicolumn{3}{|l|}{ DES } & \multicolumn{3}{|c|}{ Tanga } \\
\hline & $\mathbf{N}^{*}$ & $\mathbf{n}$ & $\%$ & $\mathbf{N}^{*}$ & $\mathbf{n}$ & $\%$ \\
\hline HIV status & 172 & & & 90 & & \\
\hline Positive & & 52 & 30.2 & & 10 & 11.1 \\
\hline Negative & & 120 & 69.8 & & 80 & 88.9 \\
\hline Hepatitis B status & 172 & & & 90 & & \\
\hline Positive & & 172 & 100.0 & & 8 & 8.9 \\
\hline Negative & & 0 & 0.0 & & 82 & 91.1 \\
\hline Syphilis & 172 & & & 90 & & \\
\hline Exposed & & 5 & 2.9 & & 0 & 0.0 \\
\hline Negative & & 167 & 97.1 & & 90 & 100.0 \\
\hline Urine chlamydia† & 179 & & & 40 & & \\
\hline Positive & & 4 & 2.2 & & 3 & 7.5 \\
\hline Negative & & 175 & 97.8 & & 37 & 92.5 \\
\hline Urine gonococcus $†$ & 179 & & & 40 & & \\
\hline Positive & & 1 & 0.6 & & 1 & 2.5 \\
\hline Negative & & 178 & 99.4 & & 39 & 97.5 \\
\hline Anal chlamydia† & 180 & & & 40 & & \\
\hline Positive & & 23 & 12.8 & & 1 & 2.5 \\
\hline Negative & & 157 & 87.2 & & 39 & 97.5 \\
\hline Anal gonococcus $†$ & & & & 40 & & \\
\hline Positive & & 26 & 14.4 & & 1 & 2.5 \\
\hline Negative & & 154 & 85.6 & & 39 & 97.5 \\
\hline Any STI (excluding HBV and HIV) & 190 & & & 91 & & \\
\hline Positive & & 45 & 23.7 & & 4 & 4.4 \\
\hline Negative & & 145 & 76.3 & & 87 & 95.6 \\
\hline
\end{tabular}

bridge to the general population, treatment in these populations is also crucial.

Of particular interest and concern is the much higher rate of rectal chlamydia and gonorrhoea than urethral infection. We suspect that as a high proportion of rectal infection is likely to be asymptomatic (only $12 \%$ in this sample reported symptoms), men do not seek treatment. This hardly differed from those without a diagnosis of rectal infection. Symptoms will be neither sensitive nor specific for a rectal STI diagnosis. Of additional concern is that the syndromic guidelines do not generally provide approaches for rectal infections. However, the fact that more than 1 in $5 \mathrm{MSM}$ in these samples had a treatable STI (and that in Dar es Salaam, more than $90 \%$ of the STIs were rectal) is a wake-up call that specific services for MSM are required in East Africa. Many of our respondents told us that if they do present for treatment, they admit only to heterosexual contact to avoid stigma, precluding any rectal investigation and diagnosis. These findings are consistent with reports of

Table $3 \mathrm{t}$ Test of difference in means for continuous variables

\begin{tabular}{lllll}
\hline Variable name & N & $\begin{array}{l}\text { HIV- } \\
\text { Mean } \pm \text { SD }\end{array}$ & $\begin{array}{l}\text { HIV+ } \\
\text { Mean } \pm \text { SD }\end{array}$ & p Value \\
\hline Number of gay men over 15 known & 252 & $11.6 \pm 12.5$ & $25.1 \pm 23.3$ & 0.0001 \\
Number of these men seen in the last month* & 260 & 5 & 10 & 0.0001 \\
Age & 262 & $25.5 \pm 5.2$ & $24.9 \pm 5.6$ & 0.5821 \\
Age had sex for the first time & 262 & $15.8 \pm 2.9$ & $14.3 \pm 3.6$ & 0.0035 \\
Lifetime number of men subject had sex with* & 258 & 6 & 15 & 0.0001 \\
Age of first sex with man & 258 & $17.4 \pm 3.6$ & $14.7 \pm 4.1$ & 0.0001 \\
PHQ9 depression score & 176 & 3 & 7 & 0.0001 \\
Internalized Homonegativity Score & 259 & $23.7 \pm 7.9$ & $20.1 \pm 8.3$ & 0.0013 \\
\hline "These variables were not normally distributed so Wilcoxon non-parametric tests were used to compare HIV+ and HIV- participants. Values \\
shown were median values instead.
\end{tabular}


Table $4 \quad \chi^{2}$ Test of association for categorical variables

\begin{tabular}{|c|c|c|c|c|}
\hline Variable name & $\mathbf{N}$ & $\begin{array}{l}\text { HIV- } \\
\%(n / N)\end{array}$ & $\begin{array}{l}\text { HIV+ } \\
\%(n / N)\end{array}$ & p Value \\
\hline Ever married/lived with female partner(Yes) & 220 & $21.3(36 / 169)$ & $11.8(6 / 51)$ & 0.1288 \\
\hline Sexual activity past 5 years (only/mostly men) & 262 & $77.0(154 / 200)$ & $95.2(59 / 62)$ & 0.0056 \\
\hline First sexual partner was a man & 262 & $41.5(83 / 200)$ & $75.8(47 / 62)$ & 0.0001 \\
\hline Been forced to have sex with someone(yes) & 260 & 33.8(67/198) & $41.9(26 / 62)$ & 0.2457 \\
\hline Paid another man for sex in the last month & 214 & $16.7(28 / 168)$ & $28.3(13 / 46)$ & 0.0767 \\
\hline Been paid to have sex in the last month & 249 & $87.2(164 / 188)$ & $88.5(54 / 61)$ & 0.7908 \\
\hline Earned income by selling sex & 262 & $28.5(57 / 200)$ & $41.9(26 / 62)$ & 0.0469 \\
\hline Currently employed (yes) & 261 & $80.4(160 / 199)$ & $83.9(52 / 62)$ & 0.5414 \\
\hline Self-identified as gay/homosexual & 252 & $49.7(95 / 191)$ & $88.5(54 / 61)$ & 0.0001 \\
\hline Used water-based lubricants & 260 & $30.7(61 / 199)$ & $63.9(39 / 61)$ & 0.0001 \\
\hline Used condom anal with recent commercial partner & 239 & $31.7(57 / 180)$ & $49.2(29 / 59)$ & 0.0152 \\
\hline Ever been tested for HIV (yes) & 259 & $77.2(153 / 198)$ & $80.3(49 / 61)$ & 0.6146 \\
\hline Depression (depressed)* & 176 & $37.1(49 / 132)$ & $68.2(30 / 44)$ & 0.0003 \\
\hline
\end{tabular}

${ }^{*}$ Depression was dichotomised at the cut-off point of 4. Depressed participants had depression score of 5 or less while non-depressed participants had depression score of 4 or less (Monahan et al, 2009 ${ }^{13}$ ).

high levels of stigma towards MSM from healthcare workers in southern Africa ${ }^{23}$ and anecdotal data from our Tanzanian sample. Although there was no question about anal transmission of STIs, nearly $95 \%$ of men knew that HIV could be transmitted by unprotected anal sex. Age at first sex was significantly higher in HIV seropositive MSM and this reinforces the need for comprehensive sexual health education during adolescence.

Significant predictors of HIV and STI infection were the size of the city and size of the gay network, both related to sexual mixing. For HIV, gay identification (with exclusively gay men more likely to be infected) was associated with infection. For STI, having unprotected RAI with one of the last 3 sexual partners was a predictor. This is not surprising given that the bulk of STI infection detected was rectal.

The relationship of STIs to HIV is not significant. This is consistent with the suggestion of Grosskurth et al, ${ }^{24}$ based on the Rakai and Mwanza studies, that despite it being known that STI treatment reduces HIV shedding in the genital tract, at a population level, the contribution of STIs to HIV transmission decreases as HIV epidemics mature. They suggest that where HIV infection becomes much more prevalent relative to other STIs, the proportion of HIV transmission attributable to co-infection with other STIs may be limited. This suggestion is consistent with the HIV epidemic in MSM in Dar es Salaam and Tanga being at a mature stage. Nevertheless, the high rates of STIs (compared with those reported in the Rakai and Mwanza studies) and the contention by Orroth et $a l^{25}$ that STIs were underestimated in the Mwanza study (which did demonstrate a reduction in HIV infection associated with STI treatment) suggest that treatment of curable STIs in MSM in cities in East Africa may still have an appreciable impact on HIV infection in this population. Study design may also have influenced our ability to show an association. If HIV infection was more distant, current STIs would

Table 5 Predictors of HIV positivity

\begin{tabular}{|c|c|c|c|c|}
\hline \multirow[b]{2}{*}{ Variable name } & \multicolumn{4}{|c|}{ HIV as dependent variable } \\
\hline & $\mathbf{N}$ & AOR & $95 \% \mathrm{Cl}$ & p Value \\
\hline STI & $199^{*}$ & 0.794 & 0.267 to 2.363 & 0.679 \\
\hline Receptive anal sex without condoms & & 1.59 & 0.624 to 4.049 & 0.3311 \\
\hline Insertive anal sex without condoms & & 0.84 & 0.282 to 2.499 & 0.7545 \\
\hline Internalized Homonegativity Score & & 0.97 & 0.918 to 1.025 & 0.279 \\
\hline City (Dar es Salaam vs Tanga) & & 3.47 & 1.255 to 9.596 & 0.0165 \\
\hline Number of gay men over 15 known & & 1.03 & 1.002 to 1.055 & 0.0322 \\
\hline Ever married or lived with a female partner & & 0.38 & 0.111 to 1.330 & 0.131 \\
\hline First sexual partner was a man & & 0.4 & 0.168 to 0.971 & 0.0428 \\
\hline Self-identified as gay/homosexual & & 3.06 & 1.007 to 9.308 & 0.0486 \\
\hline
\end{tabular}


Table 6 Predictors of STI

\begin{tabular}{|c|c|c|c|c|}
\hline \multirow[b]{2}{*}{ Variable name } & \multicolumn{4}{|c|}{ STI as dependent variable } \\
\hline & $\mathbf{N}$ & AOR & $95 \% \mathrm{Cl}$ & p Value \\
\hline HIV status & $198^{*}$ & 0.951 & 0.375 to 2.407 & 0.915 \\
\hline Receptive anal sex without condoms & & 3.69 & 1.471 to 9.254 & 0.0054 \\
\hline Insertive anal sex without condoms & & 0.908 & 0.353 to 2.339 & 0.842 \\
\hline City (Dar es Salaam vs Tanga) & & 8.217 & 2.519 to 26.809 & 0.0005 \\
\hline Number of gay men over 15 known & & 1.03 & 0.996 to 1.064 & 0.0836 \\
\hline Number of gay men seen in the last 1 month & & 0.96 & 0.916 to 0.998 & 0.038 \\
\hline Ever been forced to have sex & & 0.54 & 0.236 to 1.228 & 0.1411 \\
\hline Age at first sex with a man & & 0.97 & 0.869 to 1.090 & 0.6415 \\
\hline Earned income selling sex & & 1.83 & 0.810 to 4.148 & 0.1457 \\
\hline
\end{tabular}

not be expected to be causally associated with HIV in these men. Prospective studies are better suited to investigating these associations. However, it is important to note that such a high rate of rectal STI demands treatment in its own right.

Limitations of the study include a relatively small sample in two cities, limiting generalisation to larger urban areas, and the fact that some $12 \%$ did not agree to STI or HIV testing which provides an unknown bias. The fact that the sample was relatively young and connected to a gay subculture suggests that it may not include many MSM peripheral to the subculture.

These data suggest that HIV rates in MSM in smaller cities will be substantially lower than in a major metropolis, with MSM in Zanzibar and Tanga (both with populations in the 200-300 000 range) having about a third the MSM HIV seroprevalence of Dar es Salaam (which is about 10 times their population size). STI rates were correspondingly lower also, suggesting that the availability of larger networks of MSM is related to infection risk. For curable STIs (which have a much shorter symptomatic infection latency than HIV but may also be asymptomatic rectally), recent unprotected RAI is a significant predictor of infection. These high rates of HIV and STI, especially rectal STIs, in MSM in Dar es Salaam and the size, complexity and distribution of the MSM community there ${ }^{8}$ underscore the urgency of developing HIV and STI risk reduction campaigns for this population in this and other large East African urban settings. Smaller provincial cities appear to have MSM communities with lower but significant HIV/STI prevalence and should also be included in HIV prevention efforts.

Acknowledgements The authors thank Erick Abdoul PA for performing the clinical examinations, Jasmine Shio and John Kashiha for conducting the data collection, Dr Vrasha Chohan for facilitating and testing the chlamydia and gonorrhoea specimens at the University of Washington/University of Nairobi Mombasa STI Laboratory, and Dr Mabula Kasubi, of the Central Pathology Laboratory at Muhimbili National Hospital, for storing the specimens.

Contributors MWR wrote the first draft of the paper, and conducted some of the statistical testing, and JN, JM, SM and RSM revised and added to the draft. RSM performed the STI laboratory testing. HOA performed the bulk of the statistical analyses and commented on the drafts.

Funding This study was funded by a grant from the US National Institute of Mental Health, 5R21MH090908 (MWR). This publication also resulted (in part) from research supported by the Baylor-UTHouston Center for AIDS Research (CFAR), an NIH funded program [AI036211].

Competing interests None.

Ethics approval The study was reviewed and approved by the University of Texas Health Science Center's Institutional Review Board (HSC-SPH-10-0033) and the Tanzanian National Institute for Medical Research (NIMR/HQ/R.8a/Nol. IX/1088)

Provenance and peer review Not commissioned; externally peer reviewed.

Data sharing statement No additional data are available.

Open Access This is an Open Access article distributed in accordance with the Creative Commons Attribution Non Commercial (CC BY-NC 4.0) license, which permits others to distribute, remix, adapt, build upon this work noncommercially, and license their derivative works on different terms, provided the original work is properly cited and the use is non-commercial. See: http:// creativecommons.org/licenses/by-nc/4.0/

\section{REFERENCES}

1. Geibel S, van der Elst EM, King'ola N, et al. "Are you on the market?": A capture-recapture enumeration of men who sell sex to men in and around Mombasa, Kenya. AIDS 2007;21:1349-54.

2. Sanders EJ, Graham SM, Okuko HS, et al. HIV-1 infection in high risk men who have sex with men in Mombasa, Kenya. AIDS 2007;21:2513-20

3. Baral S, Trapence G, Motimedi F, et al. HIV prevalence, risks for HIV infection, and human rights among men who have sex with men (MSM) in Malawi, Namibia, and Botswana. PLOS ONE 2009;4: e4997.

4. Price MA, Wasima R, Mwangome $M$, et al. Identifying at-risk populations in Kenya and South Africa: HIV incidence in cohorts of men who report sex with men, sex workers, and youth. J Acquir Immune Defic Syndr 2012;59:185-93.

5. Sanders EJ, Okuku HS, Smith AD, et al. High HIV incidence, correlates of HIV-1 acquisition, and high viral loads following seroconversion among MSM. AIDS 2013;27:437-46.

6. Dahoma M, Johnston LG, Hoplman A, et al. HIV and related risk behavior among men who have sex with men in Zanzibar, Tanzania: results of a behavioral surveillance survey. AIDS Behav 2011;15:186-92.

7. Johnston LG, Holman A, Dahoma M, et al. HIV risk and the overlap of injecting drug use and high-risk sexual behaviours among men who have sex with men in Zanzibar (Unguja), Tanzania. Int J Drug Pol 2010;21:485-92. 
8. Ross MW, Nyoni J, Bowen AM, et al. Sexual and geographic organisation of men who have sex with men in a large East African city: opportunities for outreach. BMJ Open 2012;2:e001813.

9. Heckathorn DD. Respondent-driven sampling: a new approach to the study of hidden populations. Soc Prob 1997;44: 174-99.

10. Smolenski DJ, Diamond PM, Ross MW, et al. Revision, criterion validity, and multi-group assessment of the Reactions to Homosexuality scale. J Pers Assess 2010;92:568-76.

11. Ross MW, Kajubi P, Mandel JS, et al. Internalized Homonegativity/ Homophobia is associated with HIV risk behaviors in Ugandan gay and bisexual men. Int J STD AIDS 2013:5:409-13.

12. Ross MW, Smolenski DJ, Kajubi P, et al. Measurement of internalized homonegativity in gay and bisexual men in Uganda: cross-cultural properties of the Internalized Homonegativity scale. Psychol Health Med 2010;15:159-65.

13. Monahan PO, Shacham E, Reece M, et al. Validity/reliability of PHQ-9 and PHQ-2 depression scales among adults living with HIV/AIDS in Western Kenya. J Gen Intern Med 2009;24:189-97.

14. Omoro SAO, Fann JR, Weymuller EA, et al. Swahili translation and validation of the Patient Health Questionnaire-9 depression scale in the Kenyan head and neck cancer patient population. Int $J$ Psychiatry Med 2006;36:367-81.

15. Heckathorn DD. Extensions of respondent-driven sampling: analyzing continuous variables and controlling for differential recruitment. Sociol Methodol 2007;37:151-208.

16. Abdul-Quader AS, Heckathorn DD, Sabin K, et al. Implementation and analysis of respondent driven sampling: lessons learned from the field. J Urban Health 2006;83(Suppl 1):1-5.

17. Liu $\mathrm{H}, \mathrm{Li} \mathrm{J}, \mathrm{Ha} \mathrm{T}$, et al. Assessment of random recruitment assumption in respondent-driven sampling in egocentric network data. Soc Netw 2012;1:13-21. http://dx.doi.org/10.423T6/sn.2012. 12002

18. Wang J, Carlson RG, Falk RS, et al. Respondent-driven sampling to recruit MDMA users: a methodological assessment. Drug Alcohol Depend 2005;78:147-57.

19. Bowring AL, Luhmann N, Pont S, et al. An urgent need to scale-up injecting drug harm services in Tanzania: Prevalence of blood-borne viruses among drug users in Temeke District, Dar es Salaam, 2011. Int J Drug Pol 2013;24:78-81.

20. Nyoni JE, Ross MW. Condom use and HIV-related behaviours in Urban Tanzanian men who have sex with men: a study of beliefs, HIV knowledge sources, and partner interactions. AIDS Care 2013;25:223-9.

21. Bui TC, Nyoni JE, Ross MW, et al. Sexual motivation, sexual transactions and sexual risk behaviors in men who have sex with men in Dar es Salaam, Tanzania. AIDS Behav 2014, in press. doi:10.1007/s10461-014-0808-x

22. Sanders EJ, Wahome E, Okuku HS, et al. Evaluation of WHO screening algorithm for the presumptive treatment of asymptomatic rectal gonorrhea and chlamydia infections in at-risk MSM in Kenya. Sex Transm Infect 2014;90:94-9.

23. Lane $\mathrm{T}$, Mogale $\mathrm{T}$, Struthers $\mathrm{H}$, et al. "They see you as a different thing": the experiences of men who have sex with men with health care workers in South African township communities. Sex Transm Infect 2008;84:430-3.

24. Grosskurth H, Gray R, Hayes R, et al. Control of sexually transmitted diseases for HIV-1 prevention: understanding the implications of the Mwanza and Rakai studies. Lancet 2000;355:1891-987.

25. Orroth KK, Korenromp EL, White RG, et al. Comparison of STD prevalences in the Mwanza, Rakai, and Masaka trial populations: the role of selection bias and diagnostic errors. Sex Transm Infect 2003;79:98-105. 